\title{
The VINON-LOCA test facility: exploring the LOCA phenomenology through an out-of-pile thermal sequence on irradiated pressurized fuel rod
}

\author{
B. Biard ${ }^{1}$, C. Colin ${ }^{1}$, S. Bernard ${ }^{1}$, V. Marty ${ }^{1}$, G. Volle ${ }^{1}$, F. Martin ${ }^{1}$, P. Charmasson ${ }^{1}$, K. Ronné ${ }^{1}$, \\ I. Moysan-Lavoine ${ }^{1}$, J.-Y. Ferrandis ${ }^{2}$, V. Schoepff ${ }^{3}$, G. Amoyal ${ }^{3}$, E. Fédérici ${ }^{1}$ \\ ${ }^{1}$ CEA, DES, IRESNE, DEC, Cadarache F-13108 Saint-Paul-Lez-Durance, France \\ ${ }^{2}$ CNRS, IES-UMR 5214, 34000, Montpellier, France \\ ${ }^{3}$ CEA, LIST, Sensors and Electronic Architectures Laboratory, CEA-Saclay, 91191 Gif-Sur-Yvette cedex, France \\ bruno.biard@cea.fr
}

\begin{abstract}
Since the out-of-pile semi-integral tests performed at Studsvik in 2011 for the NRC [1] and the Halden Loss-Of-Coolant Accident (LOCA) test series IFA650 [2], a major safety interest has raised for Fuel Fragmentation, Relocation and Dispersal (FFRD) during a LOCA sequence. In addition to the characteristics of the fuel ejected from the rod after the clad failure, the fuel behaviour before the clad failure is still to be investigated, especially its fragmentation and its possible relocation within the rod during the clad ballooning phase. Furthermore, the chronology and the sequencing of these phenomena is of particular interest.
\end{abstract}

For this purpose, the VINON-LOCA program, lying in the framework of a trilateral agreement between EDF, Framatome and CEA, is aimed at performing Out-Of-Pile heating tests on irradiated repressurized fuel rods, reproducing a typical Loss Of Coolant Accident thermal sequence. The VINON-LOCA experimental set-up is located in the so-called VERDON lab of the LECA-STAR hot cell complex. This lab was dedicated to the VERDON-ISTP program [3]. The VINON-LOCA set-up is thus largely instrumented for addressing not only these FFRD topics, but also Fission Gas Release (FGR), combining both online measurement (gamma stations, gamma camera, acoustic sensor, pressure, temperatures, flow meters, microGC...), and pre- and post-test characterization (gamma scanning, tomography, metrology, fuel fragments weighing and sieving, gas analyses...).

An extensive and substantial qualification campaign has been performed to validate the furnace design regarding the desired test conditions, and to qualify the instrumentation. Following some preliminary modelling and calculations, it has included tests on an out-of-cell twin mockup and tests on dummy inactive rods in the hot cell. This allowed achieving successfully the first experimental qualification test of the program end of 2019 on an irradiated $\mathrm{UO}_{2}$ fuel rodlet. A second irradiated experiment is planned with increased instrumentation capabilities, notably a 2D gamma camera for online fuel motion detection.

Keywords - Nuclear fuel / experimentation / gamma imaging / FFRD / LOCA / Hot lab.

\section{INTRODUCTION}

A loss-of-coolant accident will impact both the cladding and the fuel of a rod. What is mainly investigated in VINON-LOCA experiments is the chronology and sequencing of the phenomena occurring during the first phase of a LOCA sequence: clad ballooning, fuel relocation, fission gas release, clad failure, fuel ejection. When do these phenomena start, and is there a specific occurrence order in between them? The topics that are addressed include thus clad behaviour, Fuel Fragmentation, Relocation and Dispersal (FFRD) and Fission Gas Release (FGR). Results are expected to provide some relevant experimental data for a better understanding of physical phenomena and for code validation, especially the ALCYONE code of the PLEIADES platform [4]. These developments are therefore conducted within a trilateral collaboration between EDF, Framatome and CEA.

The LOCA-type sequence that has been chosen (Fig. 1) starts from a conditioning plateau at $300^{\circ} \mathrm{C}$, under inert atmosphere (argon circulation), and follows a desired thermal ramp at $5^{\circ} \mathrm{C} / \mathrm{s}$, up to a maximal possible temperature of $1200^{\circ} \mathrm{C}$. The absence of external cooling leads to an increase of the rod inner temperature and pressure (in case of a closed volume), up to, eventually, the clad ballooning and failure, which should occur between 800 and $1000^{\circ} \mathrm{C}$. The experimental sequence will then be ended by a natural cooling.

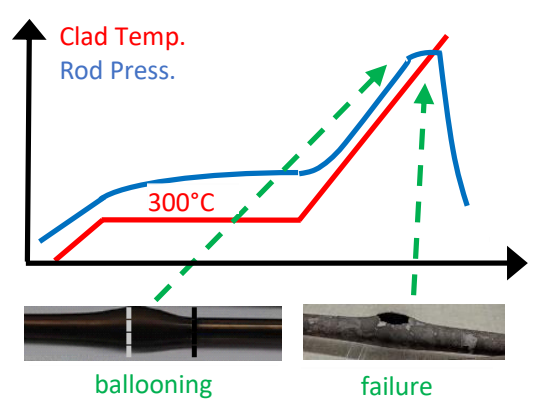

Fig. 1. LOCA sequence and clad expected behaviour. 
The rods to be tested are refabricated from rods irradiated in power plant, shortened to approximately $20 \mathrm{~cm}$ of fissile column height, and with a maximum burn-up of $90 \mathrm{GWd} / \mathrm{t}$ (limitations due to furnace dimension and safety authorization for the facility). Two Zircaloy-4 half-pellets are placed at each side of the fissile stack, and the rod is equipped with two welded plugs. The upper plug is axially drilled so as to connect the inner free volume of the rod to an extended plenum instrumented with a pressure sensor. Before the test, the test rod is pressurized at a pressure representative of End-Of-Life conditions (typically 50 bar at ambient conditions), either with pure $\mathrm{He}$ or with a $\mathrm{He} / \mathrm{Ar}$ calibrated mix.

\section{FACILITY AND INSTRUMENTATION}

The LECA-STAR fuel hot cell complex, located in the CEA Cadarache Research Center, welcomes the VERDON lab comprising two adjacent hot cells, a glove box and their associated utilities. This lab initially hosted the VERDON-ISTP program [3], and VINON-LOCA takes benefits from the equipment dedicated to this previous program.

A High Frequency inductive furnace generates heat in a tungsten susceptor tube, providing indirect heating to the test rod (Fig. 2). The HF generator was already available, but a specific inductive coil, helicoidal plate shape, has been designed and qualified. The rod is held in its upper part and centered in the furnace, in the susceptor tube, by its instrumented line through a metal piece ensuring the furnace air tightness and the crossing for the thermocouples attached to the cladding (rather than welded) for monitoring the clad surface temperature.
The instrumentation and the furnace configuration are illustrated on Fig. 2.

Several gamma stations are available with High Purity Germanium (HPGe) detectors:

- The Furnace Sighting looks online at the rod center for detecting fuel motion in the expected ballooning and failure region through ${ }^{137} \mathrm{Cs}$ activity measurement.

- The Plenum Sighting looks at the plenum cavity (empty Zircaloy cladding tube), following the fission gas release before clad failure through ${ }^{85} \mathrm{Kr}$ activity measurement.

- The Gas Sighting focuses at the experimental circuit downwards the furnace, following the fission gas release and transport after clad failure, once again through ${ }^{85} \mathrm{Kr}$ activity measurement.

These gamma examination systems will be complemented by a new 2D gamma camera (Fig. 3), which is currently under implementation in the hot cell. This camera will record the fuel motion in the same ballooning region as the Furnace gamma sighting, but at a higher speed and in two dimensions (creating online gamma mapping instead of a mean local measurement). This fast gamma imaging system has been specifically designed for the VINON-LOCA needs (detector choice, collimator design, mechanical integration...) in collaboration with CEA/LIST at Saclay [5]. The 2D active panel is an ADVACAM WidePix sensor with a detection window size of $28.2 \times 71.5 \mathrm{~mm}$ composed of $512 \times 1280$ pixels of $55 \times 55 \mu \mathrm{m}$. This sensor, cooled by water circulation, is placed behind a $6 \mathrm{~cm}-$ thick tungsten collimator comprising approximatively 500 cylindrical holes of $1 \mathrm{~mm}$ diameter. A circular rail support allows the azimuthal positioning around the rod in the furnace. The acquisition rate for VINON-LOCA will be around 10 to 50 frames/s.

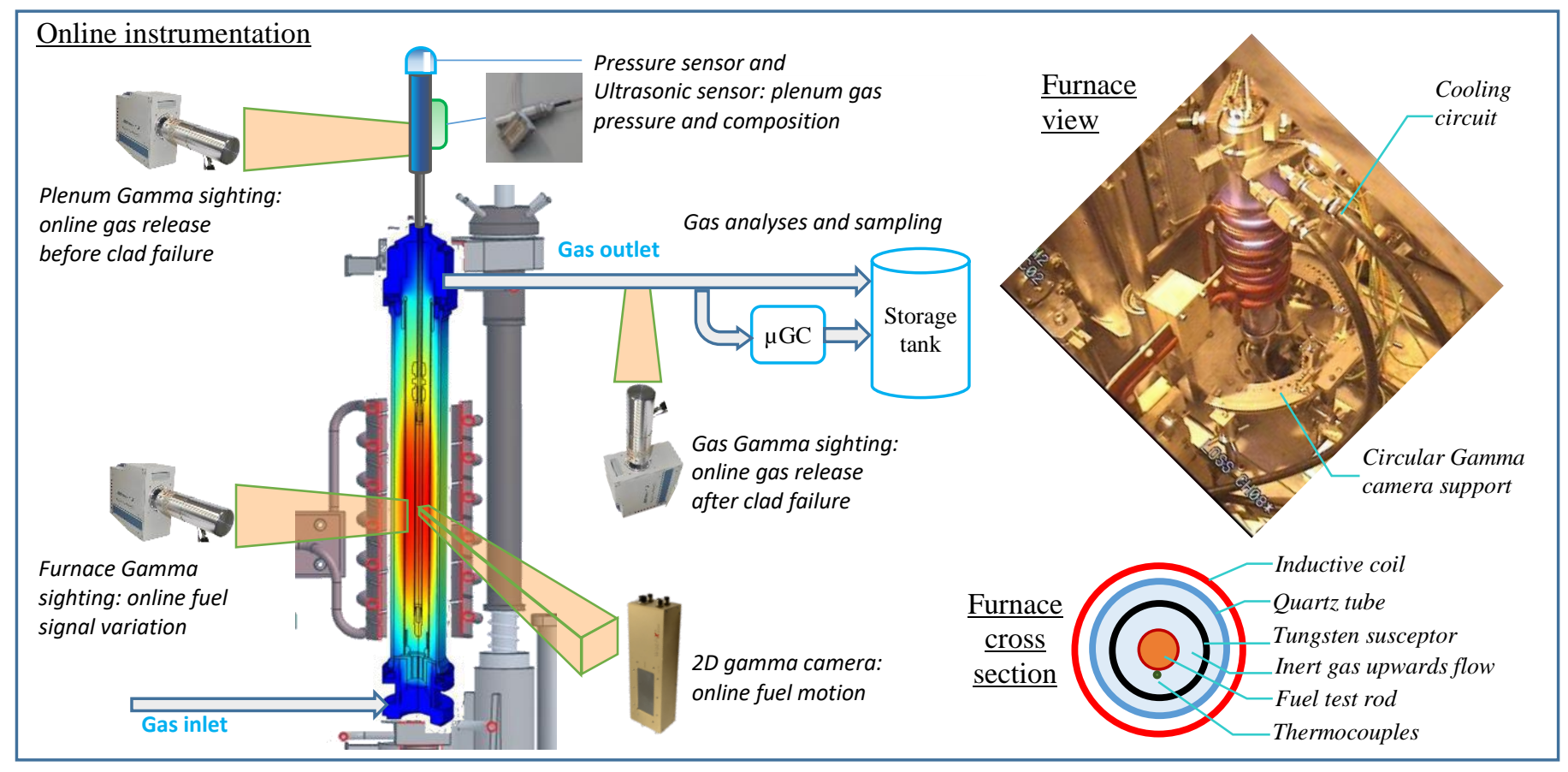

Fig. 2. VINON-LOCA online instrumentation, furnace view and furnace cross section 


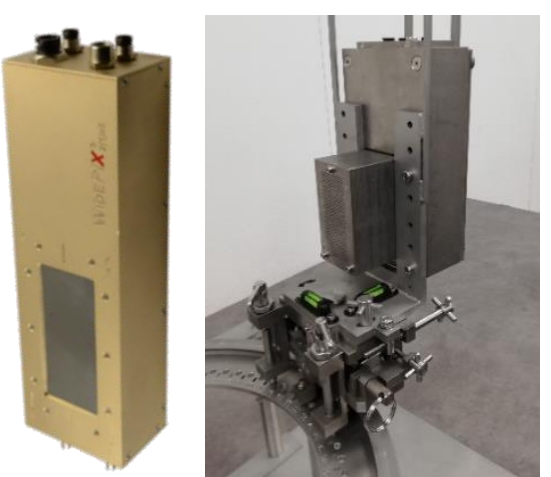

Fig. 3. 2D gamma imaging camera. Left: Advacam WidePix sensor. Right: sensor housed in the collimation box and mounted on its alignment support.

In addition to the clad thermocouples and to the gamma systems, the rod inner pressure is recorded in the plenum cavity by a piezoelectric pressure sensor couples to a Pt100 temperature sensor. It monitors the rod pressure evolution during the thermal ramp and detects the onset of ballooning and clad failure.

The plenum cavity is also equipped with an acoustic ultrasonic sensor, developed and patented by IES/CNRS and EDF [6] and fabricated by Sonaxis. This sensor estimates the gas composition through sound speed measurement, until the clad fails and the pressure becomes too low (Fig. 4). The sensor is stuck onto the cavity with an acoustic coupling material for ensuring proper sound wave propagation. The HORTUS software, specifically developed by IES for this experimentation, ensures on-line measurement and analysis. Post-acquisition analyses based on other set of parameters can be performed, depending on the measurement aim: estimating the $\mathrm{Xe}$ fraction within $\mathrm{He}$, or the fraction of a mix $\mathrm{Xe} / \mathrm{Kr}$ within He for example.
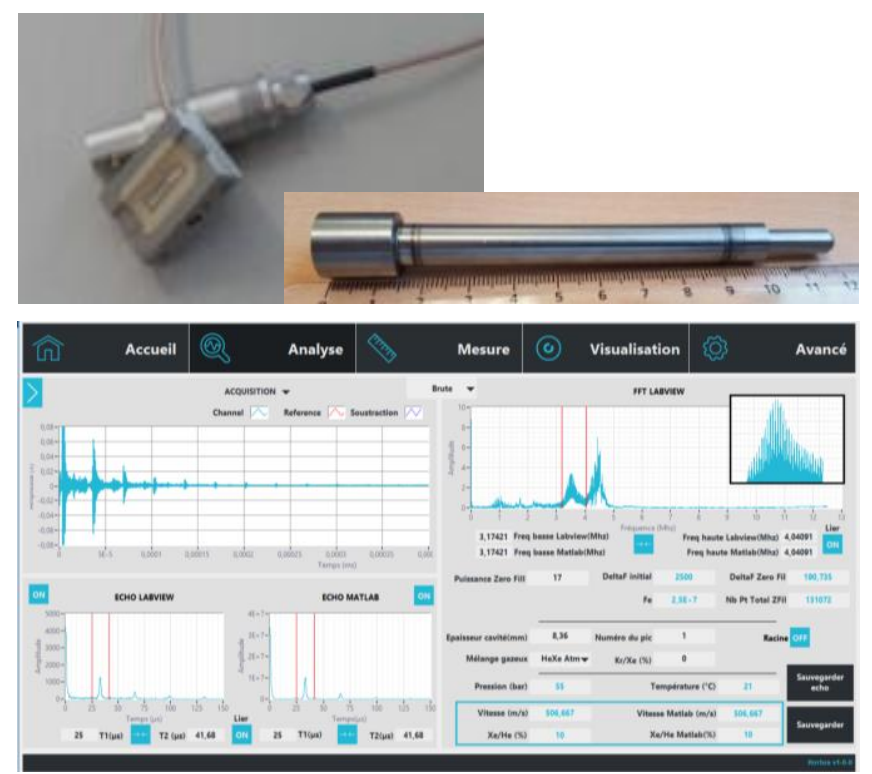

Fig. 4. Ultrasonic sensor. Top left: the sensor and its connector. Top right: the plenum cavity on which the sensor is stuck. Bottom: the associated HORTUS software (example of measurement on a cavity filled with a $10 \% \mathrm{Xe}$ in He standard gas mixture at 55bar and room temperature).
During the test, a laminar flow of inert gas (classically argon at a $60 \mathrm{sccm}$ flowrate) circulates upward in the furnace around the fuel rod, and is guided through a high efficiency Poral filter to a glove box outside the hot cell. The latter comprises a micro gas chromatograph and a storage tank. Once the hydraulic conditions are established, the thermal sequence starts with a relatively slow heating up to $300^{\circ} \mathrm{C}$ at $1{ }^{\circ} \mathrm{C} / \mathrm{s}$. A conditioning plateau lasts between 20 to 30 minutes in order to reach a correct thermal equilibrium for the furnace and its components, and for the test sample and its instrumented line. This plateau is also intended for a final checking of the instrumentation, and for acquiring the initial state of the test rod (2D gamma image, gamma emission level, thermal profile). The final ramp is then performed from $300^{\circ} \mathrm{C}$ either to a desired pre-defined value (e.g. $1000^{\circ} \mathrm{C}$, up to $1200^{\circ} \mathrm{C}$ ) or to the rod failure (test stopped manually based on the rod inner pressure measurement). The heating is then cut and the thermal sequence is generally terminated by a free cooling, although it could be followed by a high temperature plateau or a controlled cooling rate.

After the rod failure, the gas circulation continues until no more radioactive krypton $\left({ }^{85} \mathrm{Kr}\right.$ isotope $)$ is detected by the Gas gamma sighting. A fraction of the circulating gas is driven to a micro gas chromatograph, for estimating the release kinetics of $\mathrm{He}, \mathrm{Kr}, \mathrm{Ar}$ or Xe. All gases are collected in a storage tank during the experiment. Post-test samplings allows quantitative gamma measurement of the ${ }^{85} \mathrm{Kr}$ total released fraction, and possible quantitative analyses.

Pre-test and post-test examinations are performed for characterizing the rod final state. First of all, before moving the failed rod out of the furnace, a gamma scanning is carried out with the Furnace gamma sighting which is mobile along the vertical axis. The $2 \mathrm{D}$ gamma camera may also acquire static images from different orientations around the rod. The failed rod is then extracted from the furnace, separated from its instrumented line and thermocouples, visually examined, and weighted for mass loss estimation. It is then transferred to the adjacent hot cell equipped with a 3-dimension examination bench (Fig. 5).

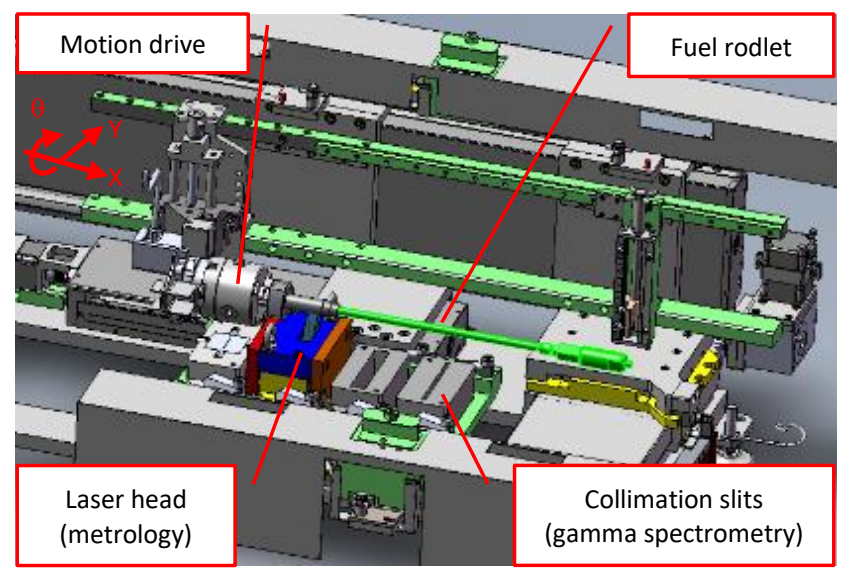

Fig. 5. 3D examination bench for gamma scanning, gamma emission tomography and laser metrology. 
The different examinations capabilities of this bench are:

- Axial gamma scanning, with adjustable collimation slits (the thinnest one being $0.6 \mathrm{~mm}$ large);

- Gamma emission tomography, with a crossed collimation slit of $0.2 \times 10 \mathrm{~mm}$ length (meaning that the gamma signal is an average on $10 \mathrm{~mm}$ along the fuel stack axis);

- Laser measurement of the clad, for estimating its diameter and deformation, notably in the balloon region.

The fuel mass balance is closed thanks to the collection and weighing of the following elements:

- the test rod itself;

- the fuel fragments ejected during the thermal sequence in the furnace, collected in the susceptor tube and in the ashtray at the furnace lower end;

- the fuel fragments recovered during the rod handling after the test (drip pans under the different equipment).

The granulometry of these fuel fragments is finally estimated by sieving.

\section{SimUlATION, QUALIFICATION AND FIRST RESULTS}

The assessment and qualification of the furnace have included modelling studies, tests in a twin mockup named EXPAT, available out of the hot cell, and finally in-cell qualification tests carried out on dummy rods. The coil design required numerous tests before retaining the final helicoidal plate configuration. The objective was to reach a rather homogeneous axial thermal gradient, optimized as a compromise between two opposite goals: on one hand the flatter the profile, the more realistic it is, but on the other hand, a peak profile is required for controlling the ballooning and failure region and positioning the online instruments. Moreover, the performances are obviously limited by the physics, and of course by the heat leaks of the system. Fig. 6 presents the final recordings of this profile, monitored with 6 thermocouples along a dummy rod cladding. Simulant pellets (Zirconia or Alumina) have been used, sometimes drilled for inserting a thermocouple. PID settings for the furnace $\mathrm{HF}$ generator were finely adjusted to reach the desired thermal gradient during the ramp. Finally, COMSOL calculations validated the thermal aspects (cooling impact at both quartz tube ends, inner rod thermal gradient for examples).

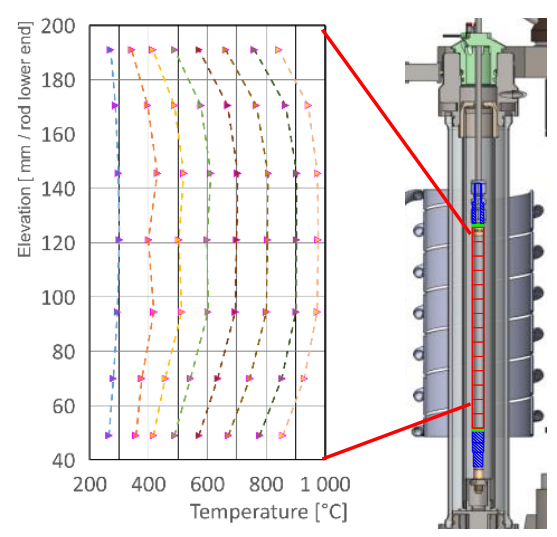

Fig. 6. Axial thermal profile in the VINON furnace (qualification test)
The qualification tests proved the capability of the VINONLOCA experimental setup and instrumentation to control and monitor the rod failure region. Many dummy rods were tested, some of them undergoing several thermal ramps, which gave to the experimenters confidence in the clad balloon and failure position. This allowed a correct alignment for the furnace gamma sighting so as to record the gamma activity signal of the fuel in this specific region. Some of these dummy rods were also tested first in the EXPAT furnace then in the VINONLOCA one to check the reproducibility of the thermal performances of both devices.

The first experimental test on irradiated $\mathrm{UO}_{2}$ was then successfully achieved by the end of 2019. Results were in good accordance with preliminary calculations performed with the CEA ALCYONE code to estimate the experimental rod behavior in such conditions. The targeted ramp was reached, the balloon and clad failure occurred at the expected location and time, and the fuel signal variation was correctly detected by the furnace gamma sighting. The ${ }^{85} \mathrm{Kr}$ gas release was properly detected both by the gas gamma sighting and by the post-test samplings, showing very consistent results. The rod was fully characterized after the test. Examples of results are shown on Fig. 7 and Fig. 8.

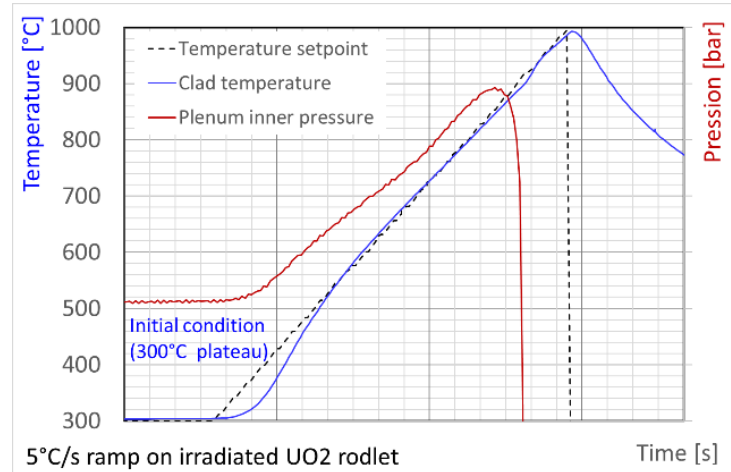

Fig. 7. Clad temperature and rod inner pressure recordings during the first test on irradiated $\mathrm{UO}_{2}$
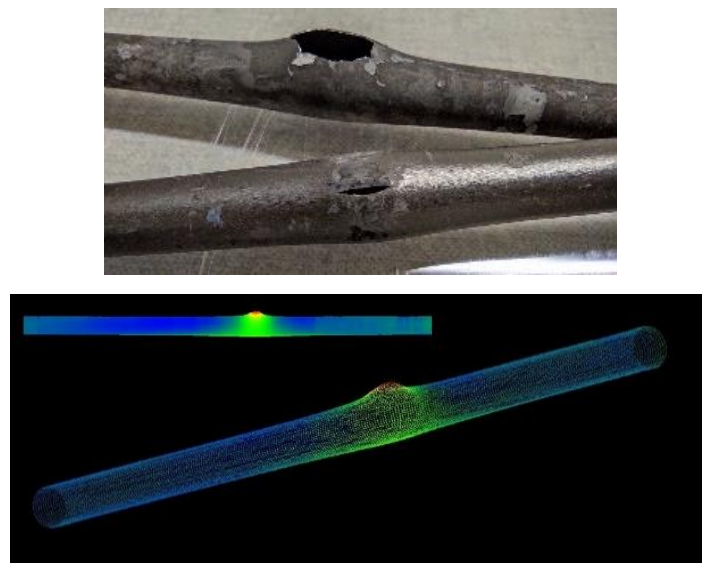

Fig. 8. Post-test examination results. Top: final aspect of burst dummy rods (pre-oxidised Zircaloy cladding, $\mathrm{Al}_{2} \mathrm{O}_{3}$ pellets, after several ramps). Bottom: 3D post-test laser metrology. 


\section{PERSPECTIVES AND CONCLUSION}

The VINON-LOCA facility has been successfully used to perform its first experimental qualification test on an irradiated $\mathrm{UO}_{2}$ fuel rodlet at the end of 2019. It will soon be equipped with enhanced instrumentation capabilities, notably the fast 2D gamma camera and upgraded electronic modules for the furnace gamma sighting. Reflections are underway for installing, if possible, other instrumentation such as a confocal imaging system for rod/clad characterization, a microphone for recording the clad failure sound (and more), or contactless pyrometric temperature measurement. Future tests to be performed in this facility might study the impact of burnup, initial pressure or free volume, various parameters of the thermal sequence (ramp stopped at clad failure or extended with a final plateau), or the behavior of innovative fuel rods (e.g. ATF) under the first phase of a LOCA transient.

\section{ACKNOWLEDGMENT}

Technical support and financial contribution of EDF and Framatome are gratefully acknowledged.

\section{REFERENCES}

[1] P. Raynaud, Fuel Fragmentation, Relocation, and Dispersal during Lossof-Coolant Accidents, US NRC, NUREG-2121, March 2012

[2] Report on Fuel Fragmentation Relocation, Dispersal, Organisation for Economic Co-operation and Development, Nuclear Energy Agency, Committee on the Safety of Nuclear Installations, NEA/CSNI/R (2016)/16

[3] A. Gallais-During, et al., Overview of the VERDON-ISTP Program and main insights from the VERDON-2 air ingress test, Annals of Nuclear Energy, Vol. 101, pp. 109-117, ISSN 0306-4549, 2017, https://doi.org/10.1016/j.anucene.2016.09.045

[4] B. Michel et al., Simulation of Pellet-Cladding Interaction with the PLEIADES Fuel Performance Software Environment, Nuclear Technology, 182:2, 124-137, 2013, DOI: 10.13182/NT13-A16424

[5] G. Amoyal et al., Design and characterization of a gamma imaging system for fuel rod deformations, ANIMMA 2021 Proceedings

[6] E. Rosenkrantz, Jean-Yves Ferrandis et al., Ultrasonic measurement of gas pressure and composition for nuclear fuel rods, Nuclear Instruments and Methods in Physics Research Section A: Accelerators, Spectrometers, Detectors and Associated Equipment, Vol. 603, Issue 3, pp. 504-509, 2009, https://doi.org/10.1016/j.nima.2009.02.028 